\title{
Fundamental Aspects of Jet Ignition for Natural Gas Engines
}

\author{
Author, co-author (Do NOT enter this information. It will be pulled from participant tab in \\ MyTechZone) \\ Affiliation (Do NOT enter this information. It will be pulled from participant tab in MyTechZone)
}

\begin{abstract}
Large-bore natural gas engines may use pre-chamber ignition. Despite extensive research in engine environments, the exact nature of the jet, as it exits the pre-chamber orifice, is not thoroughly understood and this leads to uncertainty in the design of such systems. In this work, a specially-designed rig comprising a quartz pre-chamber fit with an orifice and a turbulent flowing mixture outside the pre-chamber was used to study the pre-chamber flame, the jet, and the subsequent premixed flame initiation mechanism by $\mathrm{OH}^{*}$ and $\mathrm{CH}^{*}$ chemiluminescence. Ethylene and methane were used. The experimental results are supplemented by LES and OD modelling, providing insights into the mass flow rate evolution at the orifice and into the nature of the fluid there. Both LES and experiment suggest that for large orifice diameters, the flow that exits the orifice is composed of a column of hot products surrounded by an annulus of unburnt pre-chamber fluid. At the interface between these layers, a cylindrical reaction zone is formed that propagates in the main chamber in the axial direction assisted by convection in the jet, but with limited propagation in the cross-stream direction. For small orifice diameters, this cylinder is too thin, and the stretch rates are too high, for a vigorous reaction zone to escape the pre-chamber, making the subsequent ignition more difficult. The methane jet flame is much weaker than the one from ethylene, consistent with the lower flame speed of methane that suggests curvature-induced quenching at the nozzle and by turbulent stretch further downstream. The velocity of the jet is too high for the ambient turbulence to influence the jet, although the latter will affect the probability of initiating the main premixed flame. The experimental and modelling results are consistent with ongoing Direct Numerical Simulations at ETH Zurich.
\end{abstract}

\section{Introduction}

Flame initiation by a turbulent jet emerging from ignition in a prechamber, often denoted as "Turbulent Jet Ignition" (TJI) in the literature, is increasingly being used in large-bore lean natural gas engines. The thorough review by Toulson et al. [1] should be consulted for most of the engine-based findings until 2010. This technology is claimed to have various advantages for high pressure, large-bore systems that are difficult to ignite by conventional electrical sparks due to the lean equivalence ratio of the mixture and the higher energy needed for breakdown. With TJI, the emergence of jets from the pre-chamber may offer multiple ignition sites for the lean premixed flame in the main chamber, which allows for quick burn rates. In addition, misfires may be avoided because the pre-

Page 1 of 10 chamber mixture may be made richer than in the main chamber by extra fuel injection and the spark can be protected from violent flow [1].

Most of the research on TJI has been performed in engine environments, where the small scales and high pressures make imaging difficult and hence the details of the process are still not fully understood. The turbulent jet ignition problem has been extensively studied in simpler rigs. Yamaguchi et al. [2] working with a divided chamber bomb and propane mixtures, investigated the ignition and burning mechanisms of the main chamber mixture by a torch jet (orifice diameters between 4 and $14 \mathrm{~mm}$ ). The nozzle diameter and the volume ratio were found to have a strong influence on the structure of the torch jet independently of the main chamber mixture ratio. Recently, Biswas et al. [3] studied the ignition mechanisms of $\mathrm{CH}_{4} /$ air and $\mathrm{H}_{2}$ /air mixtures and, similarly to Yamaguchi et al., two ignition mechanisms were identified that were called "jet ignition" and "flame ignition", corresponding to jets consisting of hot combustion products and wrinkled turbulent flames, respectively. They observed that the ignition took place at the side surface of the hot jet during the deceleration process and that increased orifice diameters or increased pressure led to the "flame ignition" regime. These authors introduced the idea of a critical global Damköhler number, as a limiting parameter that separates the ignition from a no-ignition regime.

Studies in rapid compression machine (RCM) have also been conducted [4-6]. Gentz et al. [4] studied the effect of nozzle diameter as well as the number of orifices. The study of the multi-nozzle configurations revealed that at near stoichiometric conditions nozzles that produce more spatially distributed jets result in faster combustion progression, while at lean conditions, nozzles of smaller diameters that produce more vigorous jets are needed to initiate combustion. Gholamisheeri et al. [5] conducted experiments in a RCM utilizing high speed imaging. Compared to Ref. [4], the authors of this study focused more on the influence of orifice diameter and mixture composition, on the jet inlet, tip speed and the rate of flame spread in the main combustion chamber. The results show that reducing the orifice diameter led to a decrease in the hot jet penetration speed.

Despite these contributions, the details of the flow and flame passage through the orifice, which seems crucial for determining overall ignition and the ignition mechanism, have not been examined extensively yet. In addition, the details of flame inside the prechamber have not been explored. The aim of the present work is to better understand the canonical problem of jet ignition through 
simplified experiments and modelling outside of the complex engine environment, in the hope that the key physical mechanisms revealed will help interpret engine observations and other experiments at realistic conditions. The experiments were performed at atmospheric pressure, and with constant atmospheric pressure in the region outside the pre-chamber, so that complications due to the rising chamber pressure in an engine are not present. This, together with the use of an optically-accessible pre-chamber, allows a clearer exploration of the structure of the ignition process, as a first step before the full engine ignition process can be understood.

\section{Methods}

\section{Experimental Methods}

\section{Apparatus}

The apparatus used is shown in Fig. 1 (upper). It consists of a quartz cylinder $32 \mathrm{~mm}$ ID and $32 \mathrm{~mm}$ long, constrained at both ends with flat, $3 \mathrm{~mm}$ thick steel plates. This volume forms the "pre-chamber". One of these plates has a hole ( $3 \mathrm{~mm}$ or $6 \mathrm{~mm})$, with sharp edges, which forms the orifice (symbol d). The other plate has an opening fit with a solenoid valve, which is used for filling the pre-chamber with the mixture to be ignited. The equivalence ratio of the mixture inside is $\phi_{1}$, usually $\phi_{1}=1$ and the fuel is ethylene, but in some experiments methane has been used. Outside the orifice, an impinging flow is used of a fully premixed air/fuel mixture of equivalence ratio $\phi_{2}$ and with steady velocity $U$ set in the range between 1 and $5 \mathrm{~m} / \mathrm{s}$. This burner was one part of the opposed jet burner of Ref. [7], and provides a turbulent flowing mixture to be ignited (once the outer flow is ignited by the jet, a flat turbulent premixed flame may be established parallel to the plate, which is the canonical stagnating premixed flame configuration). Although this is not an enclosed flow, we may loosely use the term "chamber" in this paper to distinguish the region outside the orifice from the "pre-chamber", which refers to the volume inside the quartz cylinder. In some experiments, $\phi_{2}=0$ so that the nature of the jet is easier to discern without the added complication of ignition of the outer fluid. Ignition is provided by a spark created by a focused $532 \mathrm{~nm}$ laser beam from a Continuum Surelite II Nd:YAG laser at $50 \mathrm{~mJ} /$ pulse. Single shot operation allows for individually controlled and timed $7 \mathrm{~ns}$ long pulses to be delivered to the cylindrical, quartz pre-chamber where a $50 \mathrm{~mm}$ focal length lens focuses the beam to about $50 \mu \mathrm{m}$ creating a spark at the center of the chamber due to local ionization of the fluid. This creates the ignition basis kernel with a toroidal shape characteristic of laser ignited mixtures [8,9].

\section{Diagnostics}

High speed chemiluminescence imaging of the $\mathrm{OH}^{*}$ and $\mathrm{CH}^{*}$ radicals were performed separately at $5.34 \mathrm{kHz}$ with a Photron SA.1 and Lavision HS-IRO high speed intensifier. The imaging system was operated with an exposure time of $183 \mu$ s and an intensifier gain of 67 . The imaging region captured both the optically accessible prechamber and the exiting jet into the ambient. Due to the large difference in signal contrast between the pre-chamber and jet chemiluminescence, an OD 0.1 neutral density filter was used to reduce chemiluminescence signal from the chamber. The imaging field-of-view was $89 \mathrm{~mm}$ wide by $102 \mathrm{~mm}$ tall with a resolution of $100 \mu \mathrm{m} /$ pixel. Various movies of $\mathrm{OH}^{*}$ and $\mathrm{CH}^{*}$ chemiluminescence for both methane and ethylene and for various ignition locations, equivalence ratios, orifice sizes, and outer flows have been collected. In this paper, a few of these results are shown in the form of extracted

Page 2 of 10 stills, with the aim to reveal the location and shape of the reaction zone at various stages of the flame development.
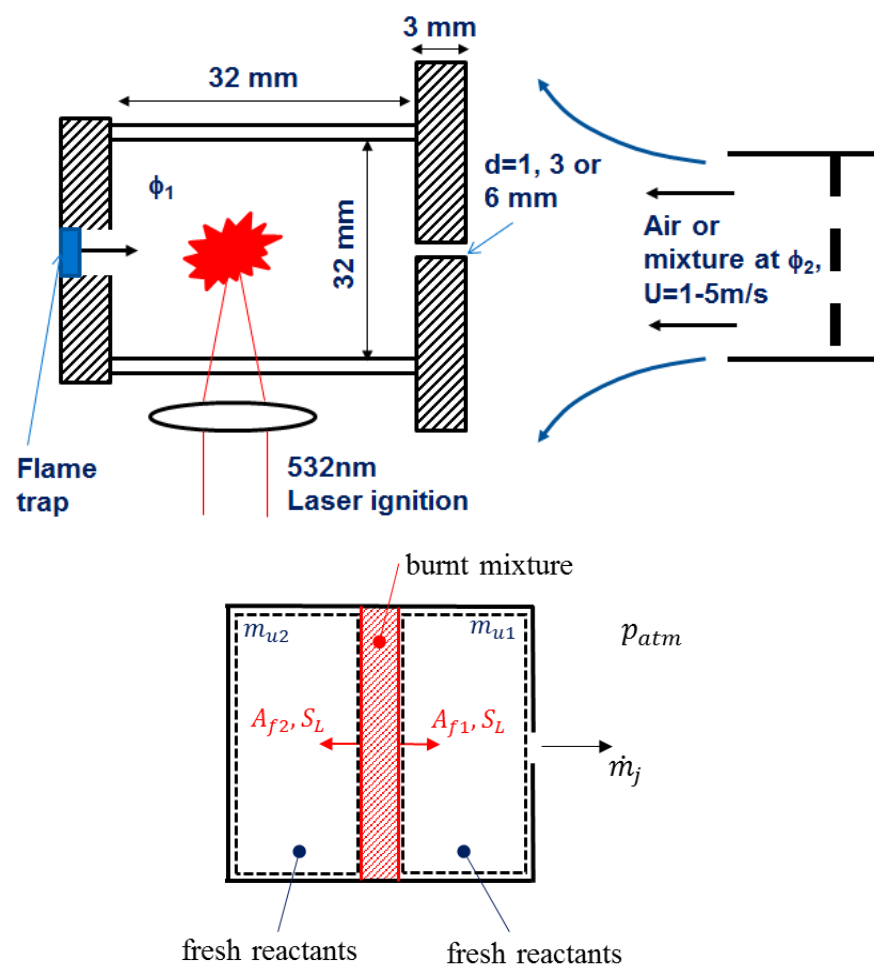

Figure 1. Upper: Sketch of the experimental rig and the flow pattern outside the pre-chamber. Lower: Definition of the thermodynamic systems used in the OD modelling.

\section{Modelling}

\section{Thermodynamic approach}

We follow an approach similar to the one proposed by Bradley and Mitchelson [10,11], who developed a system of volume-averaged governing equations for the pressure and mass inside an explosion vessel fit with a burst diaphragm, equivalent to treating it as a control volume with mass escaping. Considering an initial mixture of fresh reactants at a given equivalence ratio, the volume inside the prechamber is divided into regions with fresh reactants and combustion products. At each time step, the generation rate of the mass of burnt mixture is computed as:

$\dot{m}_{b}=\rho_{u} A_{f} S_{l}$

where $\rho_{u}$ is the density of the unburnt mixture, $A_{f}$ is the flame surface area and $S_{L}$ is the laminar flame speed. The latter is computed using a correlation that allows the dependence on the pressure and temperature to be taken into account [12]. The computation of the flame surface area is one of the most important modelling aspects and the overall accuracy of the model strongly depends on the assumptions made for its computation. The flame shape can be either assumed a-priori (for example spherical flame shape as is done in Ref. [10]) or derived from CFD simulations. For the sake of simplicity, in this work a planar flame normal to the axis of the nozzle and initially located at a distance $x_{F}$ (ignition location) from the back of the pre-chamber is considered. Two propagating fronts 
depart from the ignition location, one moving towards the nozzle and the other one in the opposite direction. In this way, the unburnt volume is divided into two different sections, separated by the flame. The two volumes of fresh reactants are considered as separate zones, as schematically shown in Fig. 1 (lower). Similar equations are applied to both zones excepting that the mass conservation of the unburnt mixture on the nozzle side has also an additional term to consider the mass flow coming out of the nozzle. The total flame area is equal to the sum of the surface area of the two flame fronts. A flame front disappears when it reaches either the nozzle or the back of the pre-chamber. The flame inside the chamber disappears when the mass of the fresh mixture is completely consumed or has exited the volume. The resulting system of equations is described next.

The mass conservation for the unburnt mixture on the two sides of the flame, with the control volume confined within the pre-chamber as indicated by the dashed lines in Fig. 1, is expressed as:

$\frac{d m_{u 1}}{d t}=-\left(\dot{m}_{j}+\dot{m}_{b 1}\right)$

$\frac{d m_{u 2}}{d t}=-\dot{m}_{b 2}$

where $\dot{m}_{j}=C_{D} \dot{m}_{i d}$ is the mass flow rate exiting the orifice, computed from the ideal flow rate for compressible flows (determined by the pressure downstream of the nozzle and the total conditions upstream) by assuming a discharge coefficient $C_{D}\left(C_{D}\right.$ equal to 0.7 has been used in this study). Ideal gas behavior is assumed in all the zones with thermodynamic properties evaluated as a function of temperature through NASA polynomials. In the unburnt mixture, transformations are assumed to be isentropic and density and temperature are computed by means of the equation of state and the isentropic relation (applied to the two unburnt zones separately):

$p \rho_{u 1,2}^{-\gamma u}=K$

where $K$ is a constant determined from the initial conditions and $\gamma$ represents the specific heat ratio. Starting from the value of the mass and density, the volume of each unburnt zone can be computed. The properties of the burnt mixture are determined by describing the combustion process as subsequent steps consisting of mixing between fresh reactants (mass determined from Eq. 1), combustion at constant volume and isentropic expansion to the pressure of the chamber. The energy released by the combustion process is computed from the lower calorific value of the fuel. A premixed mixture with a given equivalence ratio, temperature and pressure equal to the value downstream of the orifice $\left(\mathrm{p}_{\mathrm{atm}}\right)$ is used as initial condition. The simulation is performed by imposing a constant time step $\Delta t$. At each time step sub-iterations are performed by imposing a pressure and computing the volume of the various zones. The convergence criterion is based on the volume of the mixture. If the total volume of the mixture (sum of the volume of burnt and unburnt zones) is not equal to the volume of the chamber the pressure is corrected until convergence is reached [10]. From the volume of the various zones and the area of the cross section, it is also possible to compute the location of the two flame fronts. The burnt mixture starts to flow out of the pre-chamber when the flame front propagating towards the nozzle reaches the orifice (i.e. no unburnt mass $m_{u 1}$ is left in the volume).

It is important to point out that more advanced models can also be built. As it will be evident from the following discussion, the shape of the flame depends on the ignition kernel and is usually far from two planar flames. Furthermore, the mixture exiting the orifice can be a mixture of both fresh reactants and products. These features can be included in a zonal model [13], however a proper calibration using experimental measurements and CFD simulations is required. Although a more detailed model is able to improve the quantitative predictions, the simplified description of the process provides useful qualitative trends.

\section{Large Eddy Simulation}

The openFOAM package (version 2.3) has been used for modelling the methane flames and for the $3 \mathrm{~mm}$ orifice. The sub-grid scale stress tensor was modelled with the constant Smagorinsky model. The combustion model proposed by Weller et al. [14] was used. This model is based on the solution of the filtered regress variable together with a transport equation for the sub-grid flame wrinkling. Although the premixed flame model used here may not fully account for flame quenching due to heat loss or high stretch, and hence may not reveal fully the details of the flow and flame at the nozzle, the simulations provide some basic flow patterns during the flame expansion process. The computational domain includes the ignition chamber and extends downstream of the orifice for about $8 \mathrm{~d}$. The domain was discretized with a hexahedral mesh of about 9.3 million cells. Adiabatic no-slip conditions were applied to solid walls; wave transmissive condition was used for the pressure at the far field boundary (downstream of the nozzle) in order to avoid reflection of pressure fluctuations.

The accuracy of the solution in the near-wall region, and, in particular along the orifice walls, is in general an important aspect to be considered for a reliable prediction of the flame evolution. In this work, a 'wall-resolved' LES approach has been used. The grid was refined close to the wall to properly capture the velocity profile in the boundary layer region. The refinement adopted in this work was found sufficient to guarantee typical values of $y+$ close to unity, with maximum values (generally associated with the development of small recirculation zones inside the orifice) lower than 10 for the entire simulated time. Phenomena related to heat transfer may also play an important role in the flame development and the adiabatic condition imposed here may not be entirely representative of the experimental configuration, especially when the flame gets closer to the wall. However, the focus of these simulations is the first stage of the flame propagation, until the combustion products reach the orifice. The effect of heat transfer at the wall will be assessed in future work.

Second-order schemes were used for spatial discretization whereas the time derivative was discretized with a first-order implicit scheme. Adjustable time step was used during the simulation to maintain the maximum Courant number lower than 0.4 in the entire domain. The resulting time step was in the range $10^{-9}-10^{-7} \mathrm{~s}$ for the time period simulated in this work. The simulation was initialised with a quiescent stoichiometric mixture of methane at atmospheric pressure. The computation of the reaction term in the regress variable equation requires the evaluation of the laminar flame speed which was computed using the correlation by Gulder [15]. Note that this correlation may need improvement at engine-relevant conditions [16]. A spark (modelled as a sink term in the regress variable equation) of duration $5 \cdot 10^{-5} \mathrm{~s}$ and diameter $5 \mathrm{~mm}$ was imposed at the beginning of the simulation to initialize the flame. No tuning of this simplified ignition treatment was attempted to match the experiment, since the focus was on the subsequent flame development process. Therefore the simulations do not reproduce exactly the experimental ignition process, but it is expected that the simulation of evolving flame can be qualitatively comparable with the experiment.

Page 3 of 10 


\section{Results and Discussion}

In the text below, a case with ethylene at $\phi_{1}=\phi_{2}=1$ and $6 \mathrm{~mm}$ orifice is described first to reveal the basic features of the phenomena during a successful ignition event of the outer flow. Following this, further details are discussed through experiments where the outer flow does not contain fuel $\left(\phi_{2}=0\right)$ and various imaging approaches $\left(\mathrm{OH}^{*}\right.$ vs. $\mathrm{CH}^{*}$ chemiluminescence), different orifice sizes, and different fuels, with the aim to provide information on the jet only. In addition, pertinent LES and OD modelling results are included in the description of the various phases of the ignition process. The movies corresponding to the experiments discussed in the paper are available from:

https://www.youtube.com/channel/UC4X7vuIYfOg7LgRBJcVZ9-g

\section{Basic features}

Figure 2 shows $\mathrm{OH}^{*}$ images at various instances for an ignition event where the outer flow contains fuel. In the beginning, the laser spark ignites a small kernel, which grows in a laminar fashion. This flame is not spherical due to the flow pattern that is immediately set-up due to the rising pressure and the pre-chamber fluid leaving the orifice. An additional reason why the flame is not spherical is due to the nature of laser ignition that promotes a toroidal shape kernel. This is caused by the interaction between the rarefaction wave and the hot gas kernel produced by the spark, and is followed by the development of a "third lobe", which then grows in the direction of the laser beam [9]. Further, as the flame grows, it eventually passes through the orifice and a jet-like $\mathrm{OH}^{*}$-emitting region is shown that grows in the axial direction. This flame probably burns the mixture that has been ejected due to flow induced by the kernel in the pre-chamber and the entrained ambient fluid that, in this case, is flammable. At the instant shown at $2.81 \mathrm{~ms}$, a spherical-like flame seems to have been initiated from the tip of the jet, while no flame propagation is evident in the cross-stream direction. This is likely due to the very high stretch rates at the sides of the jet and the direction of the entrainment stream that brings the ambient fluid inwards towards the axis. At the last instant shown, the outer flame is growing securely, but the inner jet flame is still visible. The evolution shown in Fig. 2 is more thoroughly analysed in the next sub-section.
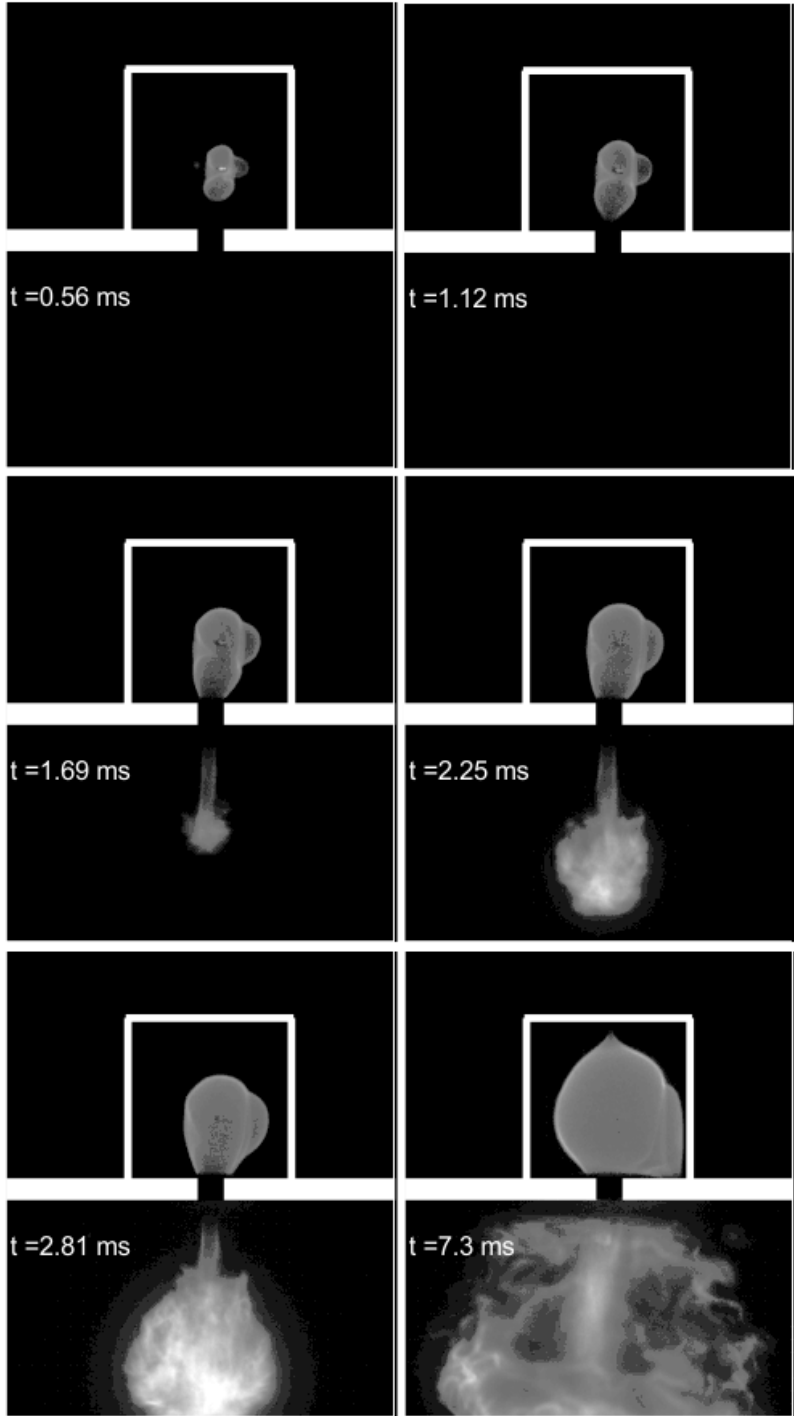

Figure 2. $\mathrm{OH}^{*}$ images at the indicated instants from laser ignition, for the $6 \mathrm{~mm}$ orifice, ethylene, $\phi_{1}=\phi_{2}=1$. Images are $102 \mathrm{~mm}$ by $89 \mathrm{~mm}$.

\section{The phases of the ignition process}

\section{Phase 1: Inner flame development}

In the experiments described here, there is no flow in the prechamber when the spark is deposited. Note that this may not be the case in real systems, where flow and turbulence inside the prechamber may be significant due to the filling process from either scavenging ports or the orifice itself as the piston moves up in the compression stroke. The present flame therefore grows in a laminarlike manner. This is evident from the $\mathrm{OH}^{*}$ images (e.g. Fig 2, Fig. 3) and from the LES simulation (Fig. 4). During this time, the pressure inside the pre-chamber has been rising due to the heat release associated with the expanding flame and hence unburnt pre-chamber fluid is leaving through the orifice to create a transient (still inert) jet into the ambient.

The LES results agree well with the experiment in this phase of the process (the simulation was performed until the flame reached the orifice, therefore only results for the first part of the flame growth are

Page 4 of 10 
available). Figure $4 \mathrm{~b}$ shows the fuel consumption in the pre-chamber evaluated as the ratio between the volume of the burnt mixture and the volume of the chamber. In the experiment, the consumed volume of reactant mixture was computed from the binarized $\mathrm{OH}^{*}$ chemiluminescence images by assuming axial symmetry, whereas in the LES it was directly computed from the resolved regress variable. Experimental results show that the rate of volume consumption increases in the first part of the flame growth, a behaviour that can be related to the increase of the surface area of the flame. However, in the second part of the flame evolution, a decrease of the rate of consumption is observed. This seems to happen after the flame has reached the orifice (Phases 2 and 3, discussed later). Many factors can contribute to this behaviour, including the evolution of the pressure inside the chamber, the variation of the jet flow rate, and quenching phenomena at the wall.

\section{Phase 2: Inner flame moving through orifice}

At the instant $0.75 \mathrm{~ms}$ in Fig. 3, and before the $3.5 \mathrm{~ms}$ instant shown in Fig 4, the flame has created a pointed edge that is moving fast towards the nozzle. The speed of this flame edge is very quick: in the snapshots in Fig. 2, the flame has travelled approximately $30 \mathrm{~mm}$ in about $0.4 \mathrm{~ms}$, giving an average speed of about $75 \mathrm{~m} / \mathrm{s}$. Similarly from the last two snapshots in Fig. 3: the flame travels about $20 \mathrm{~mm}$ in about $0.2 \mathrm{~ms}$, giving an even larger average speed. This is too quick for normal turbulent premixed flame propagation: this flame edge travels fast due to the convection by the escaping flow, which is due to the rising pressure difference between the pre- and the main chamber.

The LES (Fig. 5) for the 3mm orifice shows that even before the flame reaches the orifice, the velocities at the orifice are quite high, $\mathrm{o}(150 \mathrm{~m} / \mathrm{s})$, and due to the dilatation, when the flame begins to move out they are even higher at the centre of the orifice. It is interesting to examine the thickness of the flame there: the transition from unburnt to burnt occurs across $0.4 \mathrm{~mm}$, which is comparable to the laminar flame thermal thickness for these conditions [17]. In addition, the fluid close to the wall of the passage is unburnt and has a finite velocity, hence demonstrating that unburnt, pre-chamber fluid is escaping through the orifice, at the outer part of the orifice, together with the flame that is located in the inner part. During this phase, some recirculation close to the wall of the passage is also observed. This is induced by the sharp edges of the orifice and tends to disappear as the jet further evolves.

The pattern of $\mathrm{OH}^{*}$ emission from the jet from the $6 \mathrm{~mm}$ orifice in the first few orifice diameters (Fig 3, $0.94 \mathrm{~ms}$ ) suggests a cylindrical reaction zone (also visible in Fig 2, $1.69 \mathrm{~ms}$ ). The thickness of the orifice plate is $3 \mathrm{~mm}$ which prevents optical access at the orifice exit and hence the images do not show conclusively if this flame is attached or lifted. The diameter of this cylindrical reaction zone is about $4 \mathrm{~mm}$, which is smaller than the $6 \mathrm{~mm}$ orifice. This is consistent with the suggestion from the simulations that surrounding this flame there is unburnt pre-chamber fluid that is moving outwards. Therefore, the jet-like flame seen immediately downstream of the orifice separates pre-chamber cold reactants and pre-chamber hot products. This is very clearly seen also in the LES (Fig. 5): close to the orifice walls, the fluid is cold, and the hot products region is a thin cylinder along the axis. The finite thickness of the flame suggests the possibility of incomplete combustion, should the two flame branches touch (i.e. if the orifice is too small). Indeed, in experiments with the $3 \mathrm{~mm}$ orifice (Fig. 8), the cylindrical reaction zone is now around $1.5 \mathrm{~mm}$ in diameter and there is less clear distinction of the two branches. For some preliminary experiments with a $1 \mathrm{~mm}$ orifice

Page 5 of 10 and a different pre-chamber of similar volume, no $\mathrm{OH}^{*}$ or $\mathrm{CH}^{*}$ emission was recorded outside the pre-chamber. In addition, switching to methane (discussed later; Fig. 10) shows a much weaker $\mathrm{OH}^{*}$ emission and no $\mathrm{CH}^{*}$ emission outside the pre-chamber.

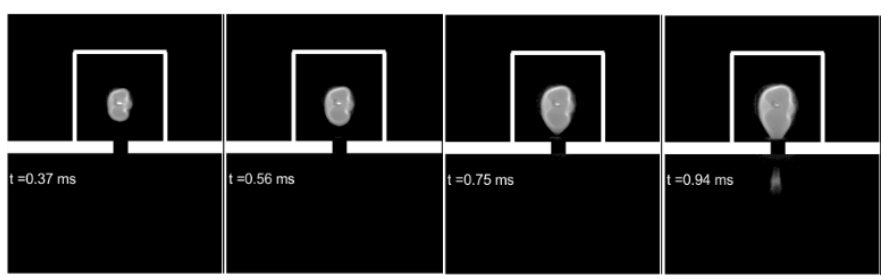

Figure $3 . \mathrm{OH}^{*}$ at the indicated instants from laser ignition, for the $6 \mathrm{~mm}$ orifice, ethylene, $\phi_{1}=1, \phi_{2}=0$. Images are $89 \mathrm{~mm}$ by $102 \mathrm{~mm}$. These images correspond to Phases 1 and 2 of the flame development process (see text for definition of the various Phases).

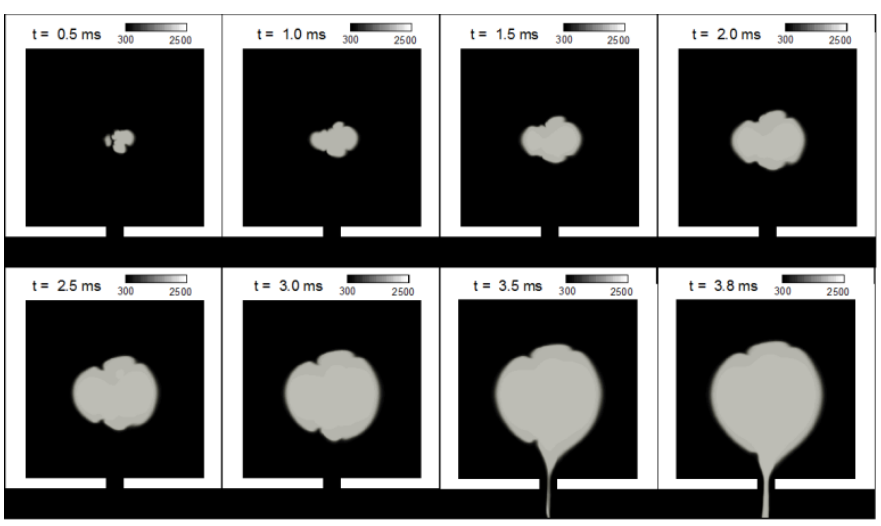

(a)

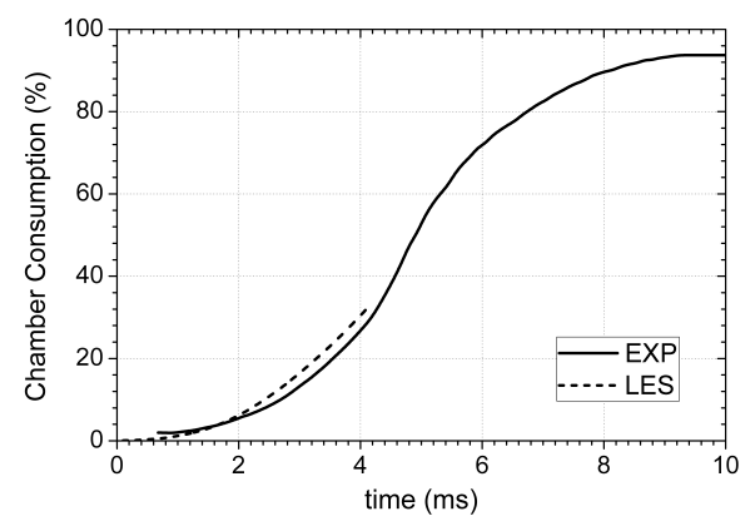

(b)

Figure 4. (a) Temperature distribution (in K) inside the pre-chamber from the LES during Phases 1 and 2 of the flame development process (see text for definition of the various Phases). (b) Comparison of burnt volume from LES and experiment. $3 \mathrm{~mm}$ orifice, methane fuel. 

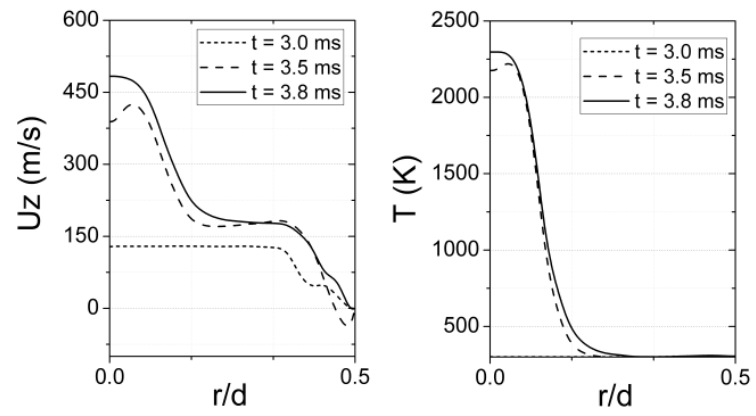

Figure 5. Velocity and temperature profiles across the orifice at various times from the spark. LES results, $3 \mathrm{~mm}$ orifice, methane fuel. Radial profiles obtained through an azimuthal average.

The above observations from the experiment and the LES suggest that the absence of flame transmission through the orifice may be associated with excessive stretch associated with large curvature, i.e. the cylindrical flame is forced to assume a diameter that is too small compared to the laminar flame thickness, rather than quenching due to heat losses at the wall. This suggestion is consistent with ongoing DNS from ETH Zurich that showed that, at the orifice, the flame is protected from the cold wall due to the fast-flowing unburnt fluid there. Although the flow inside the passage has very large shear rates $\left(\mathrm{o}\left(10^{5}\right) \mathrm{s}^{-1}\right.$; Fig. 5), the flame inside the passage does not experience correspondingly high aerodynamic strain rates because it is parallel to the flow. This alignment may explain why the flame may survive through the orifice despite the very large velocity gradients. However, outside the orifice, the stretch rate on the flame due to aerodynamic strain can be higher due to an angle development between the flame and the flow and due to the developing turbulence in the transient jet and the possibility of extinction cannot be discounted.

\section{Phase 3: Jet flame development}

Once the flame has passed through the orifice, it stays cylinder-like for a few jet diameters. In the case of the $6 \mathrm{~mm}$ nozzle (Figs. 2, 6, 7), the flame front propagates into unburnt mixture and gives a visible large reacting region that is moving with the jet; this is the main initiation site of the ambient flame (Fig. 2) if the ambience contains fuel. Once this flame has passed, the pre-chamber flame is still growing, unburnt and burnt fluid are still being pumped out, and hence a cylindrical flame with a closed end downstream is set-up and has a quasi-steady flame in the shape of a pointed arch lasting for a few ms. The length of this flame is about 5-10 orifice diameters, for both orifices tested, and is clearly seen in the case of no fuel in the ambient fluid (Figs. 6,7,8). The $\mathrm{CH}^{*}$ chemiluminescence (Fig. 7) marks this quasi-steady flame clearer than the $\mathrm{OH}^{*}$ (Fig. 6). However, switching to a weaker fuel (methane; Figs 9-10) shows that this jet flame is shorter and there is no $\mathrm{CH}^{*}$, which suggests a partially-quenched flame.

\section{Phase 4: Outer flame ignition}

The tip of the transient jet (Fig. 2, 2.25 and $2.8 \mathrm{~ms}$ ) provides the main ignition site. Note that the ignition of the ambient fluid, in this case, comes from the flame that propagated first into the pre-chamber fluid that had already escaped the nozzle. In an engine with a scavenged system, this mixture is usually richer than the main chamber mixture, Page 6 of 10 and hence the burn-out of the pre-chamber fluid outside the prechamber seems a very important first stage of the main chamber flame ignition process. Even in the absence of outer fuel (Figs. 6,7), there is a large transient flame propagating into this mixture with the jet. The main flame ignition has never been observed to start from the sides of the jet, in the first few orifice diameters, likely due to the high velocities and stretch rates there. In the case of the smaller orifice (Fig. 8), there is no evidence of such flame travelling down the jet; only the obelisk-like flame appears. This is also the case for methane (Figs. 9 and 10): only a weak $\mathrm{OH}^{*}$ jet-like flame appears, with no bulge at the tip. The absence of this flame may be the reason why in the present experiments, ignition of the outer flow with the methane was much more difficult compared to ethylene.

\section{Phase 5: Jet decay}

Once the flame in the pre-chamber has grown fully, the pressure differential between pre- and main chamber falls, and the jet flame decays. The loss of $\mathrm{OH}^{*}$ and $\mathrm{CH}^{*}$ emission outside the pre-chamber seems to coincide with the complete coverage of the pre-chamber with flame. This suggests that throughout the ignition transient, the jet flame outside the orifice is sustained by unburnt fluid coming out of the pre-chamber, since in the experiments shown in Figs. 6-10 there is no fuel in the main chamber. Even for the case of fuel in the main chamber, Fig. 2, the last instant shown $(7.3 \mathrm{~ms})$ includes a cylindrical jet flame that is still visible inside the spherical expanding main chamber flame that has probably consumed the fuel outside the orifice.

The $3 \mathrm{~mm}$ orifice produces an interesting phenomenon: towards the end of the process, but still lasting significant time (Fig. 8, see $5.43 \mathrm{~ms}$ image), the emission from the post-flame region inside the pre-chamber eventually begins to get stronger with time, rather than decaying, and for a while a bead-like pattern is seen in the jet. The continuous jet flame is destroyed and patches of $\mathrm{OH}^{*}$ emission are interlaced with regions of no emission. However, this structure eventually decays once there is no more heat generation in the prechamber.

Note that the whole process, from laser ignition to complete prechamber burnout, lasts around $10 \mathrm{~ms}$. Given that the characteristic dimension of the pre-chamber is $32 \mathrm{~mm}$ and assuming a central ignition so that the flame travels one half of that, the speed of the flame is $6.4 \mathrm{~m} / \mathrm{s}$. Given a volumetric expansion ratio of about 7.5 suggests an average displacement speed of $0.85 \mathrm{~m} / \mathrm{s}$, which is not far from the laminar burning velocity of stoichiometric ethylene [17]. 

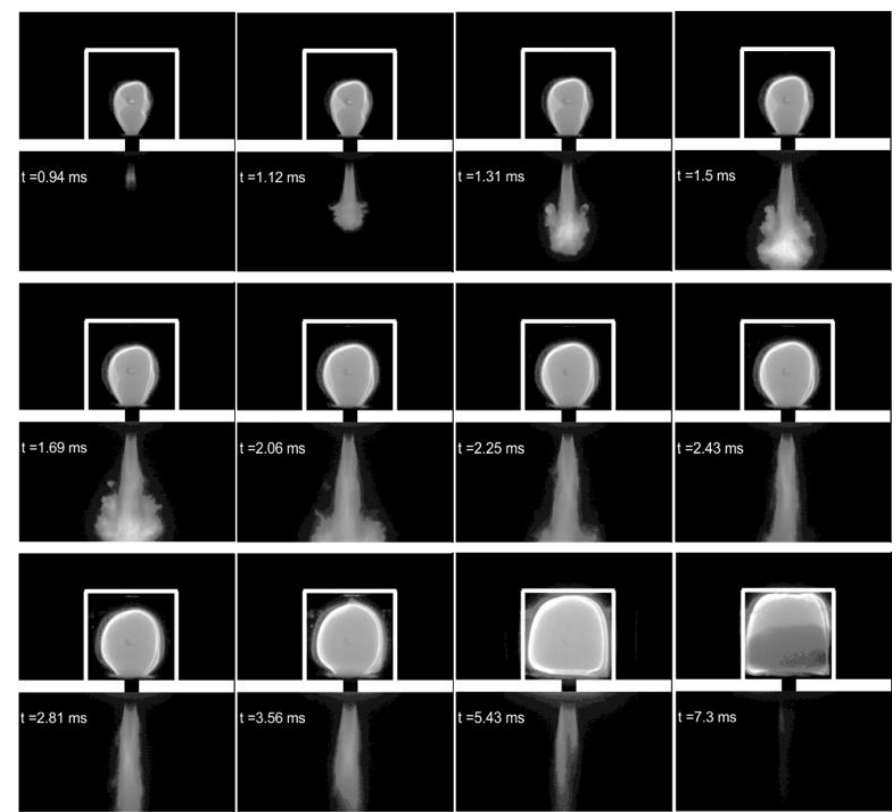

Figure $6 . \mathrm{OH}^{*}$ at the indicated instants from laser ignition, for the $6 \mathrm{~mm}$ orifice, ethylene, $\phi_{1}=1, \phi_{2}=0$. Images are $89 \mathrm{~mm}$ by $102 \mathrm{~mm}$.

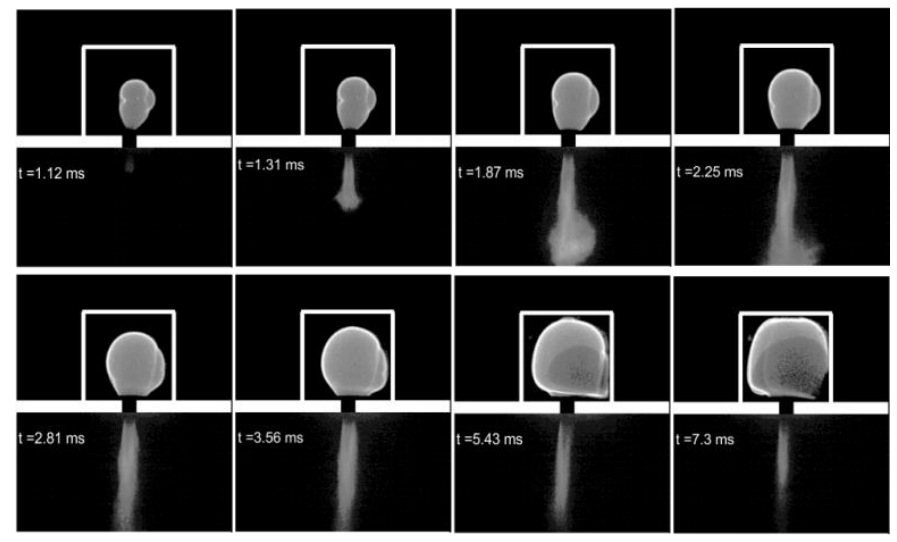

Figure 7. $\mathrm{CH}^{*}$ at the indicated instants from laser ignition, for the $6 \mathrm{~mm}$ orifice, ethylene, $\phi_{1}=1, \phi_{2}=0$. Images are $89 \mathrm{~mm}$ by $102 \mathrm{~mm}$.

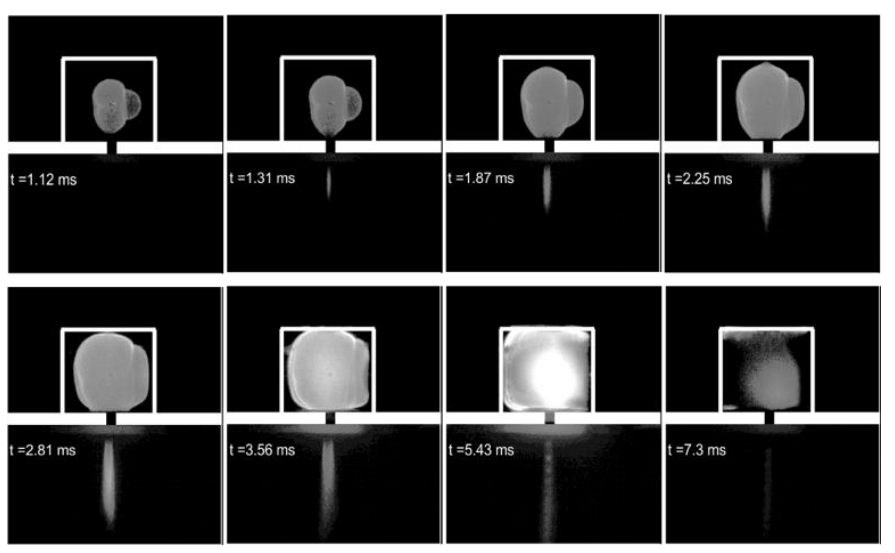

Figure $8 . \mathrm{OH}^{*}$ (top 2 rows) at the indicated instants from laser ignition, for the $3 \mathrm{~mm}$ orifice, ethylene, $\phi_{1}=1, \phi_{2}=0$. Images are $89 \mathrm{~mm}$ by $102 \mathrm{~mm}$.
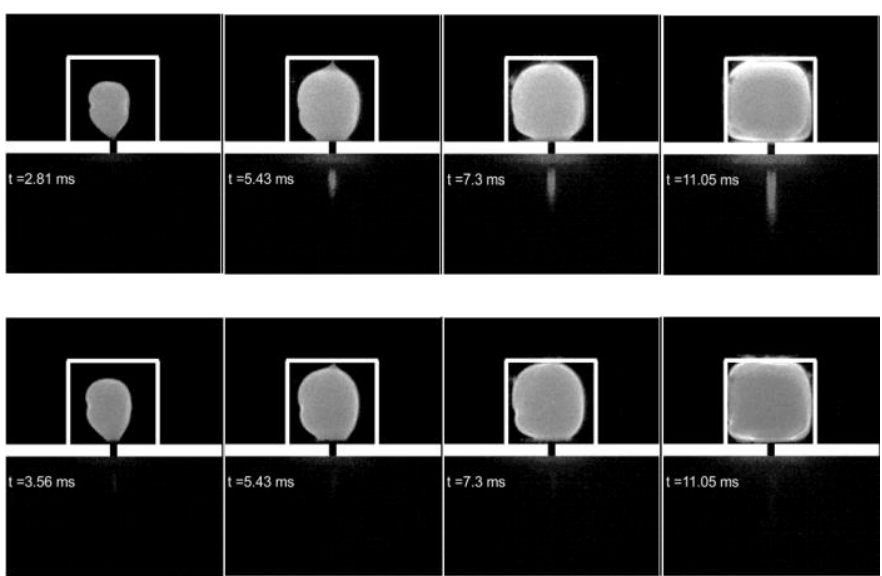

Figure 9. $\mathrm{OH}^{*}$ (upper) and $\mathrm{CH}^{*}$ (lower) at the indicated instants from laser ignition, for the $3 \mathrm{~mm}$ orifice, methane, $\phi_{1}=1, \phi_{2}=0$. Images are $89 \mathrm{~mm}$ by 102 $\mathrm{mm}$. The two measurements were not simultaneous.

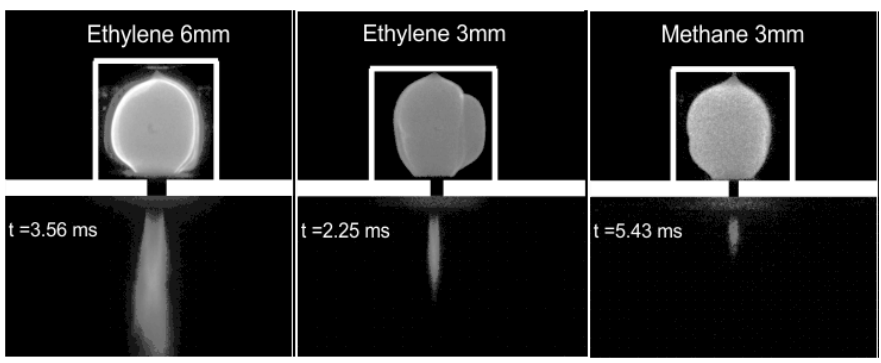

Figure 10. Comparison of $\mathrm{OH}^{*}$ at the instant of approximately $75 \%$ prechamber burnout for the different cases studied, $\phi_{1}=1, \phi_{2}=0$. Images are $89 \mathrm{~mm}$ by $102 \mathrm{~mm}$.

\section{Comparison of fuels}

In the experiments presented so far, stoichiometric ethylene was used in the pre-chamber. The flame speed of $\mathrm{C}_{2} \mathrm{H}_{4}$ at atmospheric conditions is about $0.8 \mathrm{~m} / \mathrm{s}$, while that of $\mathrm{CH}_{4}$ is about $0.4 \mathrm{~m} / \mathrm{s}$ [17]. The flame thickness is proportionally thicker for methane. This 
suggests that a jet ignition process with methane will be different than with ethylene due to the increased propensity to quench at the orifice. Figure 9 shows instances of flame growth from an experiment with methane in the pre-chamber. It is evident from the $\mathrm{OH}^{*}$ emission that the pointer arch-shaped jet flame is shorter and thinner in $\mathrm{CH}_{4}$ compared to the $\mathrm{C}_{2} \mathrm{H}_{4}$ case (see also Fig. 10 for a direct comparison), but also that there is no visible $\mathrm{CH}^{*}$ emission outside the orifice. This is an important observation that suggests that the reaction zone outside the orifice is fully or partially quenched in the case of $\mathrm{CH}_{4}$.

Assuming decay laws for a well-developed self-preserving steady axisymmetric turbulent jet, the magnitude of the turbulent velocity fluctuations at the centerline along the axis at a distance $x$ from the nozzle may be estimated as $\mathrm{u}^{\prime} \approx 0.2 \mathrm{U}(x)$, and the integral lengthscale as $0.1 x$ [18], which for a $3 \mathrm{~mm}$ nozzle and with initial jet velocity 200 $\mathrm{m} / \mathrm{s}$, at $x=10 \mathrm{~d}$ gives a Karlovitz number defined by $\mathrm{Ka}=0.25\left(\mathrm{u}^{\prime} / \mathrm{S}_{\mathrm{L}}\right)^{2} \mathrm{Re}_{\mathrm{t}}{ }^{-1 / 2}$ [19] equal to 3.2 for ethylene, but 13.2 for methane. The latter is above the critical value of about 9 evaluated for the present fuels [19]. Hence, following a conventional turbulent premixed flame extinction criterion derived mostly from studies of spark ignition in homogeneous mixtures in isotropic turbulence, the methane flame may fail due to high turbulent stretch at the developed region of the jet, while the ethylene flame is not expected to, consistently with the present observations.

\section{Two-zone modelling results}

Although the approach used to model the combustion in the two-zone model is simplified compared to the flame structure observed in both experiments and LES computations, this tool can be used to give a first estimate of the global quantities inside the chamber. Figure 11 shows the pressure in the ignitor chamber predicted by the two-zone model. The pressure reaches a maximum at the time instant when the flame front gets to the nozzle, and then decreases. The maximum pressure is higher for the case with smaller diameter whereas the time required to the flame front to reach the nozzle increases as the diameter decreases. In addition, the mass flow rate out is higher by about factor of two for the $6 \mathrm{~mm}$ orifice compared to the $3 \mathrm{~mm}$ orifice, which suggests that the area-averaged velocity is about half, which in turn suggests lower turbulent velocity fluctuations and hence stretch rate in the pre-chamber fluid jet that is likely to promote ignition. After the flame reaches the orifice, there is continuous ejection of hot products from the nozzle from both orifices for a significant time. These trends are consistent with the experimental observations. The computation can be refined by supplying to the model the time history of the flame area (for example extracted from the LES computation) and the mass fraction of unburnt mixture at the nozzle exit after Phase 2. This will be attempted in future work.
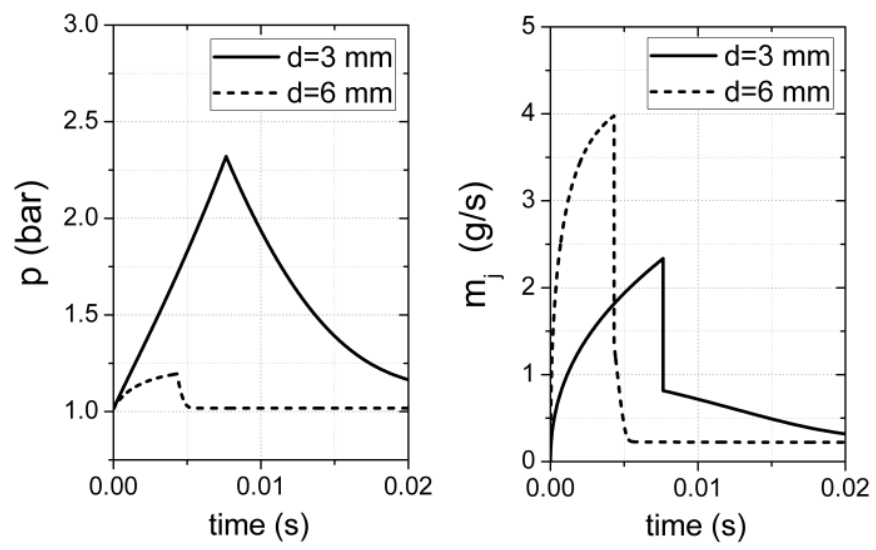

Figure 11. Results from the 0D model. Pressure and jet mass flow rate predicted for two different nozzle diameters.

\section{Further discussion}

Following the above presentation of experimental and modelling results, the following overall picture of the jet flame ignition mechanism may be put together. Figure 12 shows schematically the various stages of the inner flame, jet flame, and outer flame evolution, consolidating the arguments put forward previously for a case where the orifice diameter, $d$, is larger than at least twice the laminar flame thickness, $\delta_{\mathrm{L}}$, hence allowing the emergence of the cylindrical reaction zone from the nozzle. The exact numerical relationship between radius of curvature of the cylindrical flame at extinction and flame thickness is not clear at present. The velocity gradients may also affect the critical radius [20] and a related phenomenon is the opening of the tip of a Bunsen flame that depends additionally on the Lewis number [17]. Nevertheless, the estimate of $2 \delta_{\mathrm{L}}$ is sufficient for the present purposes as a scaling argument. In the case $\left(2 \delta_{\mathrm{L}} / \mathrm{d}\right)<1$, the continuous reaction sheet emerging from the orifice allows the development at the head of the jet of a turbulent flame brush propagating quickly into the pre-chamber / main chamber fluid mixture and once this transient ends, a sizeable ignition kernel has been created that allows main chamber flame initiation and propagation. In the case $\left(2 \delta_{\mathrm{L}} / \mathrm{d}\right)>1$, Fig 13 , which is close to the case of $3 \mathrm{~mm}$ orifice with methane, there is no reaction zone through the nozzle, which then transmits only hot products (fully- or partially-burnt). This hot-products jet may then lead to ignition as it mixes with the already-ejected pre-chamber fluid and the main chamber mixture, however this ignition method is expected to lead to a lower ignition probability. Modelling of this problem for laminar [20] and turbulent [21,22] jets has been attempted, but critical conditions for ignition have not been extensively developed yet.

The mechanisms proposed here are consistent with the observations of Ref. [3] who categorized ignition broadly as "jet" or "flame", but Figs 12 and 13 provide more detail into the underlying phenomena and additionally discuss the nature of the quenching process at the orifice.

Page 8 of 10 

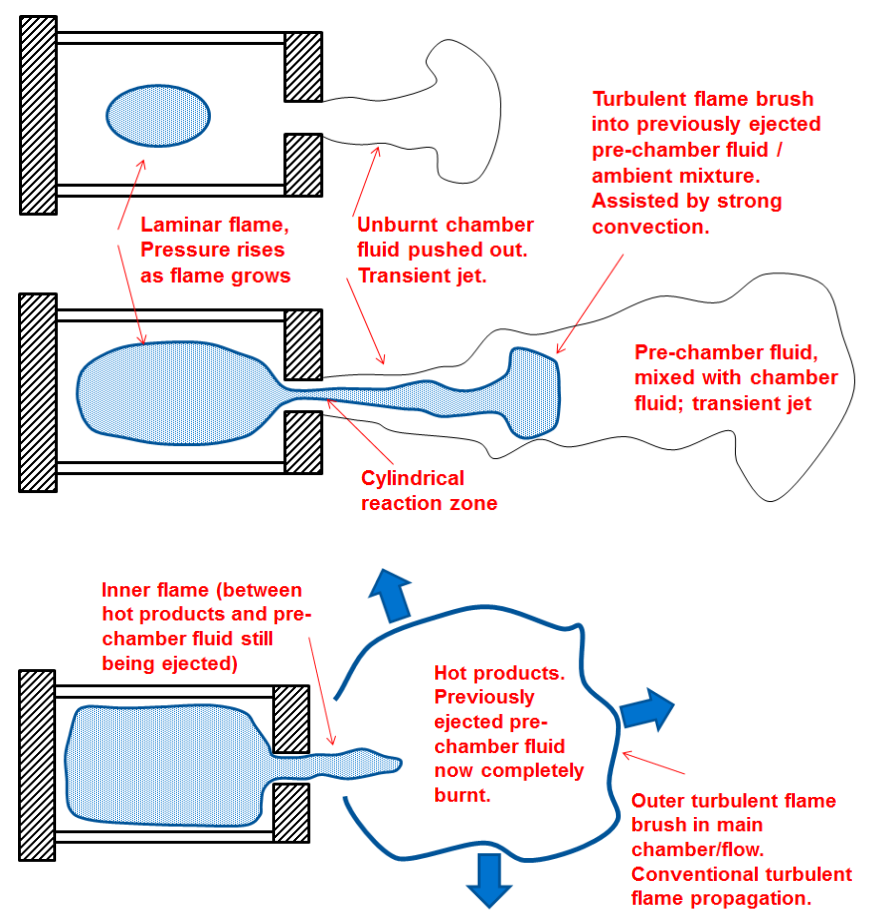

Figure 12. Schematic presentation of the jet ignition development process, for the case $\mathrm{d}>2 \delta_{\mathrm{L}}$, based on the current results (experimental, LES, 0D) and ongoing DNS results from ETH. Upper: early times (Phase 1); Middle: Flame through orifice (Phases 2 and 3); Lower: Late times (Phase 4).

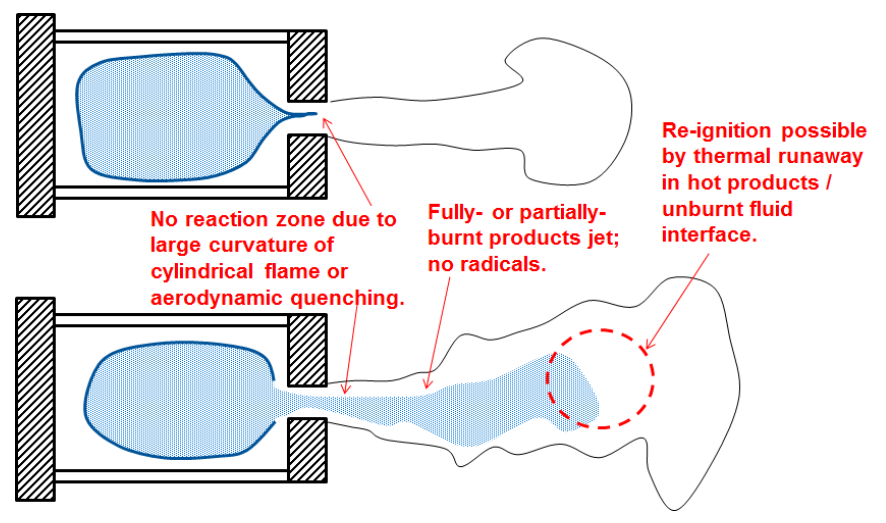

Figure 13. Schematic presentation of the jet ignition development process, for the case $\mathrm{d}<2 \delta_{\mathrm{L}}$, based on the current results (experimental, LES) and ongoing DNS results from ETH. Phases 2 and 3 .

There are many complicating factors that may limit the applicability of these results to an engine. First, concerning the pre-chamber design and conditions, the location of the ignitor in the pre-chamber will affect the pressure rise and the instant the flame reaches the nozzle [23]. These can alter the pre-chamber fluid jet that is being formed, and hence the equivalence ratio distribution in the immediate vicinity of nozzle in the main chamber. In addition, the higher volume-to-nozzle area, the higher the pressure difference between pre-chamber and main-chamber, and this increases the jet momentum which makes the jet flame more prone to extinction. The turbulence in the pre-chamber can also affect rate of flame speed and pressure rise in the pre-chamber and hence affect the jet momentum. The timing of the flame arrival at the nozzle relative to the overall pre-

Page 9 of 10 chamber burn time affects both the amount of pre-chamber fluid that is ejected and the duration of Phase 3, which keeps supporting the main chamber ignition process. The exact geometrical shape of the orifice will affect the diameter and stretch of the cylindrical flame escaping the orifice, and may hence affect the chances of ignition. Second, concerning the coupling with the main engine chamber, as the jet grows and the main chamber ignites, or even as the piston still moves, the pressure differential may be evolving, and hence the flow pattern can change, slowing down the jet development. In addition, jet impingement on the piston or walls may affect the downstream motion of the reaction zone into the pre-chamber fluid jet, hence affecting overall ignition success by large-scale transport effects. Despite these complications, and of course the fact that the high pressure and temperature in an engine make the flame quicker and thinner and ignition times shorter, the present results highlight some details of the jet ignition process that are relevant and were not available previously, and that warrant further investigation with both simulation [24,25] and experiment. Direct comparisons of this ignition mechanism with the dual-fuel (pilot ignition) system are also available [26] and they can be supplemented with the present results. The Large-Eddy Simulations may also be improved with better correlations for flame speed [27] if engine conditions are targeted.

\section{Conclusions}

The mechanism of turbulent jet ignition has been further elucidated in this work by studying an optically-accessible pre-chamber under atmospheric conditions and imaging the flame by $\mathrm{OH}^{*}$ and $\mathrm{CH}^{*}$ chemiluminescence. Ethylene and methane were used to explore effects of reactivity. The configuration has also been studied by LES and a thermodynamic model. Both LES and experiment suggest that for large orifice diameters, the flow that exits the orifice is composed of a column of hot products surrounded by an annulus of unburnt prechamber fluid that is being ejected from the pre-chamber due to the rising pressure inside the pre-chamber. At the interface between these layers, a cylindrical reaction zone is formed that propagates in the main chamber in the axial direction assisted by convection in the jet, but with limited propagation in the cross-stream direction. For small orifice diameters, this cylindrical flame is too thin, and the stretch rates are too high, for a vigorous reaction zone to emerge from the pre-chamber, making the subsequent ignition more difficult. The methane jet flame is much weaker than the one from ethylene, consistent with the lower flame speed of methane that suggests curvature-induced quenching at the nozzle and by turbulent stretch further downstream.

\section{References}

1. Toulson, E., Schock, A.J., Attard,W.P. "A review of prechamber initiated jet ignition combustion systems", SAE Paper 2010-01-2263, 2010.

2. S. Yamaguchi, Ohiwa, N. and Hasegawa, T. "Ignition and Burning Process in a Divided Chamber Bomb," Combustion and Flame 59: 177-187, 1985.

3. S. Biswas, S. Tanvir, H. Wang, and L. Qiao, "On ignition mechanisms of premixed CH4/air and H2/air using a hot turbulent jet generated by pre-chamber combustion", Applied Thermal Engineering 106: 925-937, 2016.

4. G. Gentz, B. Thelen, M. Gholamisheeri, P. Litke, A. Brown, J. Hoke, and E. Toulson, "A study of the influence of orifice diameter on a turbulent jet ignition system through combustion visualization and performance characterization in a rapid 
compression machine," Applied Thermal Engineering 81:399411, 2015.

5. M. Gholamisheeri, B. C. Thelen, G. R. Gentz, I. S. Wichman, and E. Toulson, "Rapid compression machine study of a premixed, variable inlet density and flow rate, confined turbulent jet," Combustion and Flame 169:321-332, 2016.

6. S. Schlatter, B. Schneider, Y.M. Wright and K. Boulouchos: "Comparative Study of Ignition Systems for Lean Burn Gas Engines in an Optically Accessible Rapid Compression Expansion Machine", SAE technical paper no. 2013-24-0112, doi: $10.4271 / 2013-24-0112$

7. S.F. Ahmed, R. Balachandran, E. Mastorakos, "Measurements of ignition probability in turbulent non-premixed counterflow flames", Proceedings of the Combustion Institute 31:1507-1513, 2007.

8. T.A. Spiglanin, A. Mcilroy, E. Fournier, R.B. Cohen, J.A. Syage, "Time-resoved imaging of flame kernels: laser spark ignition of $\mathrm{H} 2 / \mathrm{O} 2 / \mathrm{Ar}$ mixtures", Combustion and Flame 102: 310-328, 1995.

9. D. Bradley, C.G.W. Sheppard, I.M. Suardjaja, R. Woolley, "Fundamentals of high-energy spark initiation with lasers", Combustion and Flame 138: 55-77, 2004.

10. Bradley, D. and Mitcheson, A., "Mathematical solutions for explosions in spherical vessels", Combustion and Flame 26:201217, 1976, doi:10.1016/0010-2180(76)90072-9.

11. Bradley, D. and Mitcheson, A., "The Venting of Gaseous Explosions in Spherical Vessels. I-theory", Combustion and Flame 32:221-236, 1978.

12. Veynante, D., Poinsot, T.J. “Theoretical and Numerical Combustion", Edwards, 2005.

13. Song, R., Gentz, G., Zhu, G., Toulson, E. and Schock, H. “A control-oriented model of turbulent jet ignition combustion in a rapid compression machine", Proceedings IMechE Part D: J Automobile Engineering, 2016. doi: $10.1177 / 0954407016670303$

14. Weller, H.G., Tabor, G., Gosman, A.D., Fureby, C., "Application of a flame-wrinkling LES combustion model to a turbulent mixing layer", Symposium (International) on Combustion, 27:889-907, 1998.

15. Gulder, O.L., "Correlations of laminar combustion data for alternative SI engine fuels", SAE Technical Paper 841000, 1984.

16. G. Xu, C. Hanauer, Y.M. Wright and K. Boulouchos: "CFDSimulation of Ignition and Combustion in Lean Burn Gas Engines", SAE Technical paper No. 2016-01-0800, doi:10.4271/2016-01-0800.

17. Law, C.K., "Combustion Physics", Cambridge University Press, 2006.

18. Tennekes, H. \& Lumley, J. L., "A first course in turbulence”, MIT Press, 1972.

19. D. Bradley, P.H. Gaskell, X.J. Gu, A. Sedaghat, "Premixed flamelet modelling: Factors influencing the turbulent heat release rate source term and the turbulent burning velocity", Combustion and Flame 143:227-245, 2005
20. Carpio, J., Inglesias, I., Vera, M. et al. "Critical radius for hot-jet ignition of hydrogen-air mixtures", International Journal of Hydrogen Energy 38:3105-3109, 2013.

21. Sadanandan, R., Markus, D., Schiessl, R. et al. "Detailed investigation of ignition by hot gas jets", Proceedings of the Combustion Institute 31:719-726, 2007.

22. Ghorbani, A., Steinhilber, G., Markus, D., Maas, U. "Ignition by transient hot turbulent jets: an investigation of ignition mechanisms by means of a PDF/REDIM method", Proceedings of the Combustion Institute 35:2191-2198, 2015.

23. Thelen, B. and Toulson, E., "A Computational Study of the Effects of Spark Location on the Performance of a Turbulent Jet Ignition System," SAE Technical Paper 2016-01-0608, 2016, doi:10.4271/2016-01-0608.

24. G. Xu; Y.M. Wright, M. Schiliro, P. Kyrtatos and K. Boulouchos: "Experimental and Numerical Investigations of the Engine Operational Conditions' Influences on a Small Passive Pre-Chamber's Behavior", under review for SAE ICE 2017.

25. G. Xu, Y.M. Wright, M. Schiliro, C. Hanauer, K. Boulouchos: "Characterization of combustion in a small un-scavenged prechamber employed in stationary gas engines", under review for Int. J. Engine Res.

26. S. Schlatter, B. Schneider, Y.M. Wright and K. Boulouchos: "Comparative Study of Ignition Systems for Lean Burn Gas Engines in an Optically Accessible Rapid Compression Expansion Machine", SAE technical paper no. 2013-24-0112, doi:10.4271/2013-24-0112

27. G. Xu, C. Hanauer, Y.M. Wright and K. Boulouchos: "CFDSimulation of Ignition and Combustion in Lean Burn Gas Engines", SAE Technical paper No. 2016-01-0800, doi:10.4271/2016-01-0800

\section{Contact Information}

Contact: E. Mastorakos, Hopkinson Lab, Engineering Department, University of Cambridge, CB2 1PZ, UK. Email: em257@eng.cam.ac.uk

\section{Acknowledgments}

At ETH, this work has been supported by the Swiss Office of Energy (grant no. SI/501133-01). At Cambridge, PMO acknowledges a scholarship from the Brazilian Government.

Page 10 of 10 